Revue d'histoire de l'Amérique française

REVUE D.HISTOIRE DE L'AMÉRIQUE FRANÇAISE

\title{
Duplessis et la Commission royale d'enquête sur les problèmes constitutionnels, 1953-1956
}

\section{René Durocher et Michèle Jean}

Volume 25, numéro 3, décembre 1971

URI : https://id.erudit.org/iderudit/303091ar

DOI : https://doi.org/10.7202/303091ar

Aller au sommaire du numéro

Éditeur(s)

Institut d'histoire de l'Amérique française

ISSN

0035-2357 (imprimé)

1492-1383 (numérique)

Découvrir la revue

Citer cet article

Durocher, R. \& Jean, M. (1971). Duplessis et la Commission royale d'enquête sur les problèmes constitutionnels, 1953-1956. Revue d'histoire de l'Amérique

française, 25(3), 337-363. https://doi.org/10.7202/303091ar d'utilisation que vous pouvez consulter en ligne. 


\title{
DUPLESSIS ET LA COMMISSION ROYALE D'ENQUETE SUR LES PROBLEMES CONSTITUTIONNELS, 1953-1956 *
}

\author{
RENÉ DUROCHER \\ York University \\ MichèLe JEAN \\ Université de Montréal
}

\section{L'impasse des années '50}

Lorsque la Commission Tremblay fut instituée en 1953, le Québec menait un combat presque désespéré contre Ottawa dans le domaine des relations fédérales-provinciales. Il essayait tant bien que mal, et plutôt mal que bien, de résister à la vague centralisatrice qui déferlait sur le Canada. Cette politique centralisatrice animée par Ottawa était d'autant plus forte qu'elle se préparait depuis les années ' 30 et qu'elle correspondait aux intérêts, aux rêves et aux ambitions de la majorité anglo-canadienne.

La guerre avait permis au gouvernement fédéral de s'affirmer comme le véritable "national government" du pays et de reléguer dans l'ombre les gouvernements provinciaux. Elle donna au gouvernement central un prestige, une autorité et un pouvoir sans précédent.

La guerre terminée, le gouvernement central refusa de se départir de son pouvoir et en particulier de son monopole sur les impôts directs que les provinces lui avait cédé en 1942 en retour de subventions. Le marché avait été des plus avantageux pour Ottawa qui, de 1941 à 1947, a perçu des impôts cédés par le Québec au montant de $\$ 2,258,381,000$. et lui a donné en compensation $\$ 102,930,372 .{ }^{1}$ Le Québec, pour des raisons pratiques évidentes et parce qu'il était convaincu que l'autonomie politique sans autonomie fiscale est vaine, refusa de renouveler les accords fiscaux de 1942 même si Ottawa lui offrait des subventions plus alléchantes. Le gouvernement fédéral, décidé à maintenir à tout prix son monopole fiscal, négocia avec chaque pro-

* Communication présentée au congrès de l'ACFAS à Sherbrooke le 16 octobre 1971.

1 Québec, Commission royale d'enquête sur les problèmes constitutionnels, Rapport de la Commission royale d'enquête sur les problèmes constitutionnels (Québec, 1956), I: 142. A l'avenir: Rapport Tremblay. 
vince individuellement. En utilisant tous les moyens à sa disposition, il réussit à convaincre ou à contraindre chacune des provinces, y compris l'Ontario qui plia l'échine en 1952. Même si le Québec était isolé et lourdement pénalisé, Duplessis persista dans son refus. ${ }^{2}$

Le gouvernement central paraissait invincible et profitant de sa position de force sur le terrain fiscal, il s'immisçait dans tous les domaines, y compris celui de l'éducation, en s'appuyant sur les analyses du rapport Massey qui complétait le célèbre rapport Rowell-Sirois.

A vrai dire, le Québec dirigé par Duplessis faisait piètre figure dans ce combat. Le désarroi des esprits était profond comme en témoigne la controverse sur les subventions fédérales aux universités. Duplessis lui-même était désemparé. C'est ainsi qu'en 1951-52, il accepta les subventions fédérales aux universités. Les explications qu'il donna pour se justifier ne peuvent faire oublier qu'il s'agissait d'une volte-face incompatible avec ses principes autonomistes. De même, lorsque la Chambre de Commerce de Montréal lui proposa d'utiliser la clause des accords fiscaux qui permettait de lever un impôt provincial de 5\% sur le revenu des particuliers sans que ceux-ci subissent une double imposition, il se moqua de cette suggestion en disant qu'il ne faisait pas de l'autonomie à $5 \% .^{3}$ Finalement, lors de la campagne électorale de 1952, on put voir un autre cas qui illustrait la confusion de Duplessis lorsqu'il déclara "que son gouvernement n'imposera jamais le revenu parce que c'est taxer le travail. Il aime mieux taxer les corporations parce que c'est une taxe sur les profits. $\gg^{4}$

L'autonomie provinciale avait été un des thèmes majeurs de l'élection de 1952 et, si l'on en juge par les résultats électoraux, il y a peut-être une indication que la population ressentait une certaine lassitude face au combat de Duplessis contre Ottawa. Les libéraux provinciaux qui, en 1948, n'avaient obtenu que 8 sièges et $38.3 \%$ des suffrages, effectuèrent en 1952 une remontée spectaculaire avec 23 sièges et $46 \%$ des votes. Chose certaine, que ce soit ou non à cause de ce que ses adversaires ont appelé

2 En principe les provinces étaient libres d'accepter ou de refuser de signer les accords fiscaux, mais en pratique, estime le Rapport Tremblay, le Québec perdit de 1947 à 1955, $\$ 136$ millions. Rapport Tremblay I: 213.

3 Entrevue avec François-Albert Angers, à Montréal le 1er septembre 1971. Voir aussi Georges-Emile Lapalme, Le vent de l'oubli (mémoires), (Montréal, 1970), II : 170-171.

${ }^{4}$ Le Devoir, 19 juin 1952. 
son autonomisme verbal et négatif, Duplessis paraissait impuissant à arrêter la politique centralisatrice d'Ottawa.

Heureusement, il y avait au Québec et particulièrement dans la région de Montréal, un groupe de nationalistes qui refusaient de capituler devant le centralisme triomphant et arrogant. Les nationalistes - qui n'étaient pas tous des admirateurs de Duplessis ni des chantres du conservatisme obtus du régime - se trouvaient notamment aux HEC, au département d'histoire de l'Université de Montréal, à Relations, au Devoir, à L'Action nationale et, assez curieusement à la Chambre de Commerce de Montréal. C'est ce mouvement nationaliste des années ' 40 et '50 qui a permis de résister à la politique brutale d'Ottawa en faveur de ce qu'on a appelé « l'intégration lucide ».

La Commission Tremblay est née des combats de ces nationalistes. Elle s'imposait pour faire le point dans les relations fédérales-provinciales et pour essayer de bâtir une argumentation et un dossier cohérents en faveur de la thèse autonomiste. Il fallait élaborer une stratégie pour sortir de l'impasse où se trouvait acculé le Québec.

\section{CREATION DE LA COMMISSION TREMBLAY}

\section{Rôle capital de la Chambre de Commerce}

Il ressort de nos recherches et des entrevues que nous avons eues avec le président de la Commission, le juge Thomas Tremblay, avec monsieur Paul-Henri Guimont et le père Richard Arès, commissaires, avec Jean-Charles Bonenfant et François-Albert Angers, experts pour la Commission, et enfin avec monsieur Roger H. Stanton, assistant-contrôleur du revenu de la Province et conseiller spécial à la Commission, ${ }^{5}$ que le rôle de la Chambre de Commerce a été déterminant dans la création de la Commission royale d'enquête sur les problèmes constitutionnels.

La Commission reconnaît elle-même ce rôle lorsqu'elle écrit que le mémoire préparé en 1947 par la Chambre de Commerce de la Cité et du District de Montréal à l'intention des deux gouvernements $«$... demeure aujourd'hui encore l'un des meilleurs que nous ayons reçus. Cette initiative endossée subséquemment par un grand nombre de corps publics et par toutes les Chambres de Commerce de la province individuellement, puis par la

5 Nous tenons à remercier ces six personnes qui ont bien voulu nous recevoir en juillet et septembre 1971. 
Fédération des Chambres de Commerce est à l'origine du mouvement d'opinion dont devait naître notre Commission. $\gg^{6}$

Ce témoignage est confirmé par François-Albert Angers qui nous a expliqué que cet intérêt de la Chambre pour les problèmes découlant des relations fédérales-provinciales remontait à la fin des années ' 30 . Il croit que c'est sous l'influence d'Esdras Minville, directeur des HEC, et expert pour la Commission Rowell-Sirois, que la Chambre s'orienta hors des problèmes purement commerciaux. Le Comité qui avait préparé ce mémoire s'était, selon Angers qui en faisait partie, efforcé de discuter sur une base non partisane. L'ingérence fédérale y était fermement dénoncée. Le document se montrait très vigoureux contre la politique d'Ottawa et faisait reposer la préservation du fédéralisme sur l'autonomie fiscale des provinces, la coordination des politiques provinciales entre elles, de la politique fédérale avec celles des provinces, le tout complété par une formule de péréquation financière. ${ }^{7}$

On y proposait le réaménagement de la fiscalité avec exclusivité provinciale en matière d'impôt sur les successions; concurrence fédérale-provinciale dans les impôts sur le revenu; perception par le gouvernement fédéral sur un rapport unique du contribuable, ainsi que des ententes temporaires et des subventions fédérales aux provinces désavantagées. ${ }^{8}$ "C'était encore loin des propositions de la Commission Tremblay, dit Angers, mais le mémoire était très sévère pour le fédéral. Il a provoqué une colère formidable d'Ottawa qui avait toujours dit: 'Que le Québec propose quelque chose et on verra. Duplessis, lui, n'avait jamais rien mis en clair !'”

De 1947 à 1952, non seulement la situation ne s'améliore pas pour le Québec, mais lorsque l'Ontario accepta les accords fiscaux en août 1952, le Québec se retrouvait tout à fait isolé, sans espoir, à l'horizon, d'une solution prochaine. C'est dans ce climat chargé de tension que se tint le Congrès de la Chambre de Commerce de la province à Ottawa du 5 au 8 octobre 1952, d'où sortit une résolution recommandant au gouvernement de la province d'instituer une commission royale d'enquête pour étudier la question de l'autonomie provinciale et des problèmes constitutionnels et fiscaux qui en découlent. ${ }^{9}$ Cette recommandation du

6 Rapport Tremblay, III, 2: 217.

7 Ibid., III, 2 : 218.

8 Ibid.

9 La Chambre de Commerce de la province de Québec, Les Relations fédérales-provinciales en matière d'impôts (novembre 1952), 36. 
Congrès devait être présentée à Duplessis par une délégation de 600 membres le 26 novembre 1952.

Cette idée, selon Angers, venait de la Chambre de Montréal. Cela coïncidait avec la présidence provinciale de Laurent Paradis, ami de Duplessis et résident de Trois-Rivières. "Après une conversation avec Duplessis, Paradis réussit à le convaincre de recevoir une délégation des Chambres de Commerce", affirme Angers qui poursuit: "Les organisateurs de la délégation rencontrèrent Duplessis dans son bureau où il aurait dit qu'il n'accorderait pas la Commission. Puis il rejoignit la délégation installée dans la salle de l'Assemblée législative où il prononça un discours. Tous avaient alors l'impression que l'affaire tournerait en queue de poisson. Mais, à la toute fin, il dit qu'il accordait la Commission." Ce revirement, selon Angers et Bonenfant, vient du fait que Duplessis fut très surpris lorsqu'il se rendit compte de la composition de la délégation qui contenait, entre autres, plusieurs sympathisants et organisateurs de l'Union Nationale.

A partir de ce moment, les choses s'accélérèrent et le 12 février 1953 la Commission était constituée. La Chambre de Commerce triomphait ! Elle écrivait, dans sa revue Commerce ${ }^{10}$ du mois d'avril 1953, un éditorial intitulé "Une "grande" enquête qui s'amorce", dans lequel elle demandait la participation de tous et chacun. Le 28 septembre de la même année, la section de Montréal tint une journée d'étude sur le mandat de la Commission à laquelle participèrent 200 représentants de différents organismes.

\section{Choix des commissaires}

Mais son rôle ne s'arrête pas là. Elle eut, toujours selon François-Albert Angers, son mot à dire dans la nomination de certains commissaires. Sur ce choix toutes les personnes que nous avons rencontrées sont d'accord pour dire que ce fut en somme un groupe assez bien structuré qui fut appelé à diriger les destinées de la Commission.

On a écrit que tous étaient des amis de Duplessis, mais il faudrait nuancer ce jugement. Le juge Thomas Tremblay, qui devait présider la Commission, explique: "Il est certain que toutes les personnes nommées étaient connues de Duplessis. Il n'aurait pas pris de chance. Par exemple, Rowat et moi-même 95-96.

10 "Une "grande" enquête qui s'amorce", Commerce, 55 (avril 1953): 
étions des amis de Duplessis, mais non des politiciens." Le juge Thomas Tremblay avait d'ailleurs une certaine expérience derrière lui puisqu'il avait déjà présidé le tribunal d'arbitrage chargé d'évaluer les biens de la Montreal Light Heat. Il avait aussi présidé l'Office d'électrification rurale et une autre commission sur le transport en commun de la ville de Montréal. Lorsque Duplessis l'a convoqué, il dit: "Je ne connais rien aux problèmes constitutionnels." Ce à quoi Maurice Duplessis répondit: “Tu l'apprendras si tu ne le sais pas." Le juge poursuit: "J'ai hésité. Mes médecins me recommandaient de ne pas accepter. On se connaissait depuis très longtemps; depuis 1927..." Puis, il accepta. Duplessis lui soumit ensuite des noms. Dans un cas, le juge Tremblay le fit changer d'idée. Mais il ne fut pas consulté sur la rédaction de la loi. Au sujet du juge Tremblay, Bonenfant affirme que "c'était un homme de gros jugement, capable de bien mener une telle entreprise. En somme, une sorte de caution pour Duplessis".

Esdras Minville, directeur de l'Ecole des Hautes Etudes commerciales et président de la Chambre de Commerce de Montréal en 1947, "... était, selon le juge, un esprit objectif et indépendant". Angers ajoute: "Duplessis ne pouvait l'éviter étant donné ses antécédents." Selon le témoignage de Jean-Charles Bonenfant, Minville comme Arès d'ailleurs, furent imposés par Montréal. Ce dernier, alors directeur-adjoint de l'Institut Social Populaire et rédacteur à la revue Relations, était, selon Angers, "assez bien vu de Duplessis".

Honoré Parent, avocat, sera lui aussi nommé commissaire. Il avait été autrefois directeur des services municipaux de la ville de Montréal. C'était un ancien président du conseil de la Chambre de Commerce de Montréal. Selon Angers, c'est à la suggestion de la Chambre qu'il fut nommé; ce qui est fort plausible. Paul-Henri Guimont, pour sa part, était un ancien des HEC. De plus, son beau-père, le notaire Trudel de TroisRivières, connaissait bien Duplessis. Il raconte lui-même: "Duplessis a dû penser que ma participation à la Commission contrarierait le père Georges-Henri Lévesque, car j'étais alors secrétaire de la faculté des sciences sociales de l'Université Laval." Enfin, le notaire John P. Rowat, président du comité protestant du Conseil de l'Instruction publique et de la Commission scolaire de Montréal, fut, semble-t-il, "l'Anglais de service”. A son sujet, les témoignages concordent pour dire qu'il n'a rien fait. Selon Bonenfant ce "ne fut pas un homme à problèmes”. Le juge Tremblay avoue qu'il fut “... plus ou moins 
intéressé. Jamais dissident". Paul-Henri Guimont souligne que Duplessis a pu penser contrôler la Commission par lui, mais en vain car "il fut un élément tout à fait passif".

Duplessis ou ses conseillers avaient tenté de maintenir un certain équilibre dans le choix des commissaires en prenant un anglophone protestant et cinq francophones catholiques. De ce nombre, quatre étaient de Montréal et deux de Québec. Chacun d'eux apportait une compétence particulière qui serait utile à la Commission. Le juge Tremblay, en plus d'être un ami et un homme de confiance de Duplessis, avait derrière lui une vaste expérience comme enquêteur tandis que Minville était un sociologue, Guimont un économiste, Arès un spécialiste des questions constitutionnelles, Rowat un spécialiste des problèmes scolaires et Parent un expert sur les questions municipales.

Bonenfant conclut à ce sujet: "Pour l'époque, ce n'était pas mal. Du moment que Duplessis acceptait le système, il devait se soumettre à certains impératifs."

\section{Mandat}

La loi créant la Commission comprenait dix 'Attendu' résumant la position constitutionnelle de la province. Elle affirmait d'abord:

Attendu que la confédération canadienne, née d'une entente entre les quatre provinces pionnières, est d'abord et surtout un pacte d'honneur entre les deux grandes races qui ont présidé à sa fondation... 11

Et plus loin:

Attendu que, depuis 1917 , le pouvoir central a envahi d'importants domaines de taxation réservés aux provinces et, par là, limité sérieusement la possibilité pour les provinces d'exercer leurs droits fiscaux dans ces domaines. ${ }^{12}$

Après cet exposé succinct de la politique autonomiste du gouvernement, on demandait à la Commission d'enquêter sur les problèmes constitutionnels, de faire rapport au lieutenantgouverneur en conseil de ses constatations et opinions et de lui soumettre ses recommandations quant aux mesures à prendre pour la sauvegarde des droits de la province, des municipalités et des corporations scolaires. On y précisait que cette Commission étudierait spécialement: a) les problèmes de la répartition

11 Rapport Tremblay, I: v.

12 Ibid. 
des impôts entre les différents ordres de gouvernement; b) les empiétements du pouvoir central dans le domaine de la taxation dirccte entre autres; c) les répercussions et les conséquences de ces empiétements; d) les problèmes constitutionnels d'ordre législatif et fiscal.

L'Enquête devait se terminer le 1er mars 1954. Trois fois le mandat dût être prolongé et le Rapport ne fut finalement remis que le 15 février 1956. C'est dire que les commissaires prirent leur rôle au sérieux. Trop au sérieux au goût de Duplessis qui croyait leur avoir confié un mandat assez restreint comprenant deux grands objectifs: les problèmes constitutionnels et la répartition des impôts.

Mais ces hommes qui avaient pour la première fois en mains un tel instrument de travail n'allaient pas se limiter comme ils le dirent à des développements peu élaborés, voire plutôt techniques. "Comment, écrivent-ils, enquêter sérieusement sur les problèmes constitutionnels sans rechercher, analyser et approfondir leS causes qui leur ont donné naissance ?" 13 Il en va de même pour l'impôt, dont ils jugèrent que l'important était d'en évaluer la répartition actuelle et d'en proposer une nouvelle si nécessaire. Ce qui supposait, selon eux “... une connaissance approfondie des besoins, $d \in \mathrm{S}$ charges, des revenus et des responsabilités de chacun des pouvoirs en cause..." ${ }^{14}$ C'est de cette conception du mandat de leur Commission qu'est sorti le volumineux Rapport Tremblay. A ce sujet, Angers affirme: "Duplessis ne voulait pas un Rapport comme ça. Il aurait voulu un travail de trois ou quatre mois. Un Rapport qui aurait démontré ce qu'il fallait..." Toutes les personnes que nous avons interviewées ont confirmé cette opinion d'Angers.

\section{Le fonctionnement de la Commission}

Les membres de la Commission rencontrèrent officiellement Dup'essis après leur nomination. Selon le juge Tremblay: "Ce fut 'bonjour, bonjour', pas beaucoup plus." Le père Arès ajoute: "Il nous dit de ne pas jeter l'argent par les fenêtres. Ce Rapport servira pour vos enfants. Pas les miens ni ceux du père Arès. Ne faites pas comme les juges qui dorment durant les sessions. Travaillez! On vous paye pour cela! Ne faites pas faire votre ouvrage par les autres !"

13 Ibid., I: 1.

14 Ibid., I: 2. 
Etait-ce là une allusion aux velléités d'engagement d'experts que pourraient avoir les commissaires ? En tout cas, il est certain que cette question suscita quelques réticences de la part du premier ministre. Le juge Tremblay se souvient que Duplessis trouvait onéreux l'engagement d'experts. "Je lui dis: si tu veux qu'on travaille, il nous faut des outils." Lorsque nous lui avons demandé s'il y eut menace de démission à certains moments, il répondit: "Non, mais j'ai dit aux commissaires: il nous faut des experts. Si Duplessis refuse, il ne nous reste qu'à prendre notre paquet et à s'en aller." Et... Duplessis accepta.

Le nom de Paul Gérin-Lajoie fut toutefois refusé. Selon Bonenfant, son nom aurait même été suggéré comme secrétaire, mais non agréé. François-Albert Angers fut accepté d'assez mauvaise grâce par Duplessis. Il dit lui-même: "Quand il annonça ma nomination, il mentionna qu'il la faisait à la demande de la Commission. Il m'a fait travailler dans des conditions épouvantables. Je ne pouvais pas engager d'assistants payés. Je recevais $\$ 5.00$ l'heure."

En ce qui a trait à cette question des fonds, le juge Tremblay mentionne qu'il y eut certaines difficultés au début et qu'ensuite tout se passa bien. Selon Paul-Henri Guimont, Duplessis fut assez mesquin sur le financement. Jean-Charles Bonenfant prétend qu'il y avait une certaine coquetterie à prouver que le tout coûterait moins cher que Rowell-Sirois. Selon le juge, les commissaires recevaient $\$ 700.00$ par mois, et lui-même $\$ 25.00$ par mois en plus de son salaire de juge. Le coût total de la Commission s'éleva à $\$ 465,658.17 .{ }^{15}$ Ce qui est très peu comparativement au coût actuel de tels projets.

Les audiences publiques de la Commission furent précédées de 34 séances d'études à huis clos. Le partage du travail “... se fit ensuite naturellement", dit le juge. Il semble que chacun ait pris ce qui l'intéressait plus particulièrement, ce qui entraîna certaines imperfections de forme dont nous reparlerons plus loin. Paul-Henri Guimont mentionne d'ailleurs que l'absence d'un secrétaire de rédaction serait la cause des défauts évidents de la rédaction finale.

On peut se demander maintenant quelle fut l'influence de Duplessis sur les travaux de la Commission et s'il intervint directement ou indirectement? A ce sujet le juge est catégorique: "Une fois qu'il avait nommé quelqu'un, il le laissait faire." Nuançant ce jugement, Paul-Henri Guimont ajoute: "J'avais

${ }^{15}$ Comptes publics de la province de Québec (1953 à 1958), passim. 
travaillé comme recherchiste pour Rowell-Sirois. Il y avait là beaucoup de latitude. On ne se sentait lié par rien du tout. A la Commission Tremblay, Duplessis nous laissa entière liberté. Il fallut toutefois essayer de contrôler une partie des travaux des experts et autres. Par exemple, nous réduisîmes les prévisions budgétaires pour les années 1956 à 1966 afin de ne pas effrayer Duplessis. En somme... il nous contrôlait mentalement !"

\section{L'impôt provincial sur le revenu des particuliers}

Cette influence s'est exercée dans les deux sens comme nous pouvons le constater au moment de la création de l'impôt provincial. Alors même que la Commission tenait des séances publiques à travers le Québec, l'Assemblée législative adoptait en février 1954 une loi qui créait pour trois ans, à compter du 1er janvier, un impôt sur le revenu des particuliers, équivalant à environ $15 \%$ de l'impôt fédéral. Ce geste très positif du gouvernement fut bien accueilli par les membres de la Commission Tremblay. Cela s'explique car, si la Commission Tremblay avait été en quelque sorte le rejeton de la Chambre de Commerce, l'impôt provincial sur le revenu des particuliers devait être celui de la Commission Tremblay.

La Commission permettait aux gens de prendre conscience du problème aigu qui se posait entre Ottawa et Québec et elle témoignait de la présence du gouvernement provincial. Les groupes et les individus demandaient au gouvernement québécois de jouer un rôle plus dynamique dans la vie de la collectivité. La plupart des gens qui comparurent devant la Commission reconnaissaient que Québec avait raison de demander une plus large part des ressources fiscales et de vouloir maintenir son autonomie. Les idées autonomistes prêchées par Duplessis et par les nationalistes depuis 1939 les avaient conditionnés à prendre cette position. Alors que, depuis la fin de la guerre, on recommandait des négociations entre Québec et Ottawa sur les questions fiscales, en novembre 1953 on peut voir la Commission Tremblay s'acheminer vers une position beaucoup plus radicale.

En effet, le 17 novembre 1953, lors de la présentation à Québec du mémoire de la Commission des Ecoles catholiques de Québec par le notaire Raymond Cossette, le juge Tremblay demanda à ce dernier :

Dans les deux dernières phrases vous dites qu'il convient que les Provinces jouissent d'une pleine liberté dans le domaine de la taxation directe et que l'intervention du Fédéral dans ce champ soit réglementée par des accords. Est-ce que vous ne 
voyez pas là l'apparence de contradiction si les Provinces sont complètement libres d'imposer des taxes directes ? Alors, pourquoi sera-t-il nécessaire de faire un accord avec le Gouvernement fédéral ? 16

Et plus tard, le même mois, soit le 23 novembre 1953, la présentation du mémoire de la ville de Port-Alfred, par Roland Fradette, c.r., procureur de cette ville, fit grande impression sur les membres de la Commission. On pouvait lire dans ce mémoire:

Elle (la ville de Port-Alfred) considère que la Province devrait affirmer ses droits de taxation, en les exerçant, afin de faire bénéficier sa population des impôts qu'elle en retirerait. Il est incontestable que le pouvoir provincial peut imposer des taxes directes pour des objets qui ressortent de sa juridiction. Les empiétements du gouvernement fédéral dans ce domaine ne devraient pas l'empêcher de recourir aux moyens de prélèvements que l'acte constitutionnel lui accorde. ${ }^{17}$

Le père Richard Arès, Paul-Henri Guimont et Roger H. Stanton nous ont tous souligné l'influence qu'avait eue sur eux ce témoignage. Au cours des conversations qui suivirent cette audience, ils proposèrent au juge d'aborder le sujet avec Duplessis. Le juge Tremblay raconte de la sorte la suite des événements:

Je veillais un soir avec Duplessis et je lui dis: la meilleure preuve qu'on tient à ses droits, c'est de les exercer. Tu vas à Ottawa et tu y proclames qu'on veut notre butin. Tes droits, tu les as. Prends l'impôt sur le revenu. Tu dis qu'Ottawa nous a volés et que cet impôt devait nous être remis après la guerre. Rien ne t'empêche de créer un loi de l'impôt sur le revenu des particuliers. Tu prouveras ainsi que tu tiens à tes droits. Tu affirmeras que la Province possède ce droit. Le moment est favorable... les gens sont éveillés. Une telle loi serait la bienvenue. Duplessis me répondit que j'étais fou et qu'il se ferait battre aux élections. J'ajoutai: Penses-y sérieusement. Le gouvernement fédéral cédera. Si tu demandes des points d'impôt, c'est Saint-Laurent qui portera le fardeau de la double taxation. C'est le temps ou jamais. Nous nous laissâmes là-dessus et deux semaines après, sans en avoir entendu parler de nouveau, j'appris que les officiers du ministère étaient en train de rédiger une loi sur l'impôt.

16 Commission royale d'enquête sur les problèmes constitutionnels, Séance tenue à Québec le 17 novembre 1953, 5: 22 .

17 Mémoire de la ville de Port-Alfred devant la Commission royale d'enquête sur les relations fédérales-provinciales et autres sujets, 1. Copio déposée à la bibliothèque des Hautes Etudes commerciales, Université de Montréal. 
Nous croyons avoir trouvé là le point de départ de cette décision restée très mystérieuse. Après sa conversation avec le juge Tremblay, Duplessis a probablement consulté d'autres personnes, subi d'autres influences que des recherches ultérieures pourront nous révéler. Mais il nous paraît maintenant clair à la suite de ces témoignages que sans l'intervention et les arguments du juge Tremblay, Duplessis n'aurait pas pris la décision audacieuse de créer unilatéralement un impôt provincial, du moins à ce moment.

Le juge Tremblay et ses collègues avaient fait une bonne évaluation de l'opinion québécoise qui se rallia autour de Duplessis. Le Rapport racontant la suite des événements mentionne que "Ce geste du gouvernement québécois reçut, en général, l'appui des contribuables, bien qu'il établît un régime de double imposition pour plus de 300,000 citoyens. Des organismes importants, comme la Chambre de Commerce du district de Montréal, adressèrent à Ottawa des mémoires demandant la pleine déduction." 18

La réponse du fédéral vint dans le discours du budget d'avril 1954. Ce qui embêtait le plus, semble-t-il, le gouvernement central c'était le préambule de la loi d'impôt qui affirmait que la province "avait priorité en matière de taxation directe". Reconnaître ce principe et reconnaître que n'importe quelle province a le droit de déterminer le montant que les habitants de cette province peuvent déduire de l'impôt apparaissait au fédéral comme inacceptable. "The demand for total deductibility of the provincial from the federal income tax could mean in an extreme case a provincial claim to the whole of the income tax collected by the federal government within the province" ${ }^{19}$, écrivait Martin P. O'Connell, dans le Canadian Forum. Cela est si vrai que c'est précisément ce que recommandera la Commission Tremblay.

Duplessis, encouragé par le juge Tremblay, avait eu l'excellente idée de prendre la population comme arbitre dans son conflit avec Ottawa. Le premier ministre Saint-Laurent releva avec fougue le défi que lui avait lancé Duplessis. Dans un célèbre discours prononcé au Club de Réforme le 18 septembre 1954, il dénonça avec véhémence la politique autonomiste de son adver-

18 Rapport Tremblay, I : 153-154.

19 Martin P. O'Connell, "Duplessis in Deflation", Canadian Forum (novembre 1954) : 171-172. Cité par C. Nish, éditeur, Quebec in the Duplessis Era, 1935-1959: Dictatorship or Democracy? (Toronto, 1970), 47. 
saire. Duplessis, le 26 septembre à Valleyfield, dans ce qui restera probablement le plus important discours de sa carrière, plaidera avec calme et éloquence la cause de l'autonomie. Cet affrontement entre les deux premiers ministres montre avec éclat que l'enjeu du conflit entre les deux gouvernements était infiniment plus qu'une question id'argent...

Finalement, chacun put se déclarer gagnant car le préambule de la loi fut modifié et la "déductibilité" de $15 \%$ devint une "réduction" de $10 \%$. Le Rapport conclut sur la question en disant: "Remarquons seulement qu'il laissait en définitive les deux gouvernements chacun sur leurs positions et ne réglait pas le problème des relations fiscales entre eux." ${ }^{20}$ La réduction de $10 \%$ laissait les deux impôts indépendants l'un de l'autre et elle s'appliquait à toutes les provinces qui imposaient ou percevaient un impôt provincial. Ceci était moins fort au plan des principes que la première formule; la réduction implique en effet une concession du fédéral, tandis que la déductibilité laissait l'initiative au provincial, ce que craignait par-dessus tout le fédéral. Malgré cela, selon François-Albert Angers, ce fut une grande décision de Duplessis: "Ce fut un geste très positif qui n'a pas eu son égal depuis ce temps. Duplessis passait à l'attaque en demandant plus. Il lançait une offensive contre Ottawa. Il allait plus loin que la Chambre de Commerce. Il chambardait tout le système."

Peut-on accepter cette appréciation de F.-A. Angers ? On pourrait, dans un premier temps, faire remarquer qu'il a fallu à Duplessis plusieurs années pour se décider puisqu'il était revenu au pouvoir en 1944. A noter aussi qu'il a dû $\in$ n fin de compte céder sur deux points majeurs: priorité et déductibilité qui, aux yeux de certains observateurs de l'époque, apparaissaient d'une extrême importance. Martin P. O'Connell estimait que si Duplessis avait gagné sur ces deux points il aurait détruit "the existing structure of tax agreements and the capacity of the federal government to discharge its function". Il ajoutait que Duplessis accepterait probablement:

... a tax agreement based on a level of deduction which will yield sums to Quebec roughly equal to what she would receive under a rental scheme... From the standpoint of Ottawa the costs would be no different, but more important, it would have gained what it deems essential - control over the level of income taxation in Quebec. If such a settlement emerges from the recent struggle the federal government will have

${ }^{20}$ Rapport Tremblay, I: 155. 
improved its ability to employ fiscal policy in its own and the national interest... 21

C'est effectivement ce que Duplessis accepta comme compromis lorsqu'il amenda sa première loi sur l'impôt. Ajoutons que le fédéral n'avait jamais nié le droit des provinces à l'impôt direct puisqu'en 1940 sept des neuf provinces canadiennes avaient un impôt sur le revenu et que même après les accords fiscaux de '47 les provinces "récalcitrantes" pouvaient lever un impôt de 5\% sans double imposition. D'une certaine manière, on aurait pu dire à Duplessis qu'il se contentait d'une autonomie à $10 \%$ !

Le parti libéral provincial, mal conseillé, voire trahi par le grand frère d'Ottawa, a tenté de soulever la population contre la double imposition s'imaginant qu'il s'agissait d'une question de "taxe". En fait, Duplessis, par cette décision unilatérale, avec comme arbitre ultime la population québécoise, venait de renverser toute la stratégie centralisatrice élaborée depuis les années '30. L'impôt provincial était la brèche qui allait permettre au Québec de faire sa révolution tranquille dans les années '60. Comme l'a si bien dit Georges-Emile Lapalme, une quinzaine d'années après ces événements, Duplessis "en se servant de l'impôt, inversait les préalables de la politique à long terme" 22 .

\section{Analyse du Rapport}

Le 15 février 1956, le premier ministre de la province de Québec recevait un document énorme contenant un million et demi de mots, 1,881 pages, soit quatre volumes très denses sans compter les onze annexes.

Les quatre volumes se subdivisent en cinq grandes parties. La première s'intitule Aperçu historique. Elle examine et fait le récit des relations fédérales-provinciales depuis la Confédération jusqu'à nos jours. La seconde partie étudie Les finances publiques: leur évolution et leur état actuel. La troisième partie s'occupe de La province de Québec et le cas canadien-français; elle étudie le problème des cultures et la place de la province de Québec dans la Confédération. La quatrième partie traite du Fédéralisme au Canada et ailleurs dans le monde. Enfin, la cinquième partie s'intitule Analyse des besoins et Recommandations. A cela s'ajoute un volume de documentation fort utile, bourré de statistiques intéressantes sur la démographie, l'éco-

21 M. O'Connell, op. cit.

22 Georges-Emile Lapalme, Le vent de l'oubli (mémoires), II: 179. 
nomie, l'enseignement, les revenus, les dépenses provinciales, etc.

On aura tout de suite vu les inconvénients d'une telle division. Elle entraîne forcément des répétitions souvent longues et fastidieuses. Ceci semble provenir du fait que chaque spécialiste d'une question a rédigé lui-même son texte qui est passé quasi intégralement dans le Rapport. Ainsi, on peut dire, si l'on se fie aux témoignages de Jean-Charles Bonenfant, de FrançoisAlbert Angers, du juge Tremblay, que le père Richard Arès a rédigé la section historique et Esdras Minville la section sur les problèmes de culture, ces deux seules parties se recoupant fréquemment. Ceci fit dire à Jean-Charles Bonenfant que le Rapport est "fabriqué comme une courtepointe". La lecture du Rapport devient de ce fait assez ardue. D'autre part, le fait que les recommandations ne soient nulle part résumées en caractères gras empêche une lecture rapide de celles-ci, lecture souvent utile à celui qui veut prendre connaissance globalement des conclusions de l'enquête. Mentionnons toutefois, à la décharge des rédacteurs, qu'il existe à la fin du second tome du troisième volume un Aperçu général et sommaire des recommandations qui résume d'une manière satisfaisante l'ensemble du Rapport.

Dans une première partie qui s'étend sur près de 175 pages, les commissaires retracent l'histoire des relations fédéralesprovinciales depuis la Confédération. Leur interprétation repose sur le postulat que la Confédération a été à la fois un pacte entre des provinces et un "pacte entre des associés égaux". Cette vision traditionnelle du fédéralisme canadien qui avait été très vivement contestée au Canada anglais dans les années trente, nous paraît très discutable, comme l'ont montré Michel Brunet et Jean-Charles Bonenfant.

“Qu'en est-il de la situation financière actuelle de la Province ?" C'est à cette question que veut répondre la deuxième partie du Rapport qui aborde l'étude "austère mais nécessaire" des Finances publiques. Cette partie contraste avec les sections socio-philosophiques du Rapport et s'avère des plus utiles à la compréhension des besoins financiers de la province à l'époque. Même si elle fait à certains moments double emploi avec d'autres composantes de l'ouvrage, cette section demeure essentielle par l'aperçu pertinent qu'elle donne des dépenses et revenus de la province, de sa dette et du budget provincial, pour finalement déboucher sur une étude comparative de ces trois derniers éléments (dépenses-revenus, dette, budget) des divers gouvernements du Canada. Ce souci d'appuyer sur une analyse solide des 
besoins économiques de la province les recommandations finales du Rapport manifeste un désir réel de ne plus se contenter de formules retentissantes et souvent très vagues.

On peut y lire, ce qui ne fut certes pas pour plaire à Duplessis, même si c'est le fédéral qu'on visait, que la province en demeurant libre et en refusant les ententes fiscales de 1947 à 1955 avait perdu $\$ 136$ millions..$^{23}$ Et les auteurs ajoutent: "Comment alors ne pas demeurer sceptique devant les déclarations répétées du gouvernement fédéral, à savoir que le régime des ententes ne touche en rien à la liberté des provinces, ..." ${ }^{24} \mathrm{Au}$ chapitre des dépenses de la province de Québec, on insiste sur le fait qu'un Etat ne peut s'administrer comme une vaste entreprise privée, genre de remarque qui n'était pas non plus de nature à plaire au premier ministre dont on connaît la traditionnelle prudence en matière de finances ! Enfin, après avoir passé en revue les revenus de la province, jugés insuffisants, on aborde une discussion sur sa dette. Celle-ci, lit-on, doit être considérée comme un moyen d'influencer la production et la distribution du revenu et non “... uniquement comme une charge qu'il faut supprimer dès que les circonstances le permettent" ${ }^{25}$. Il y est démontré que la politique très conservatrice de la province lui a nui à plusieurs points de vue. En résumé, nous pouvons dire que cette partie, surtout descriptive, apparaît comme l'une des plus solides du Rapport.

\section{Problèmes de culture et fédéralisme}

Dans la troisième et la quatrième parties, qui étudient les problèmes de culture et le fédéralisme, si les auteurs nous présentent le Canada français comme une nation au sens sociologique du terme (communauté de culture, de langue, etc.), ils ne manquent pas de faire certaines mises en garde contre le nationalisme politique. Les principaux caractères de la culture canadienne-française en feraient une culture chrétienne, spiritualiste, personnaliste, communautaire, qualitative, centrée sur l'homme, ordonné à son perfectionnement, à la réalisation intégrale de sa double vocation naturelle et surnaturelle. ${ }^{26}$

Après avoir souligné qu'on ne doit pas nécessairement confondre Etat et Nation, ils expriment certaines réticences vis-à-vis

${ }^{23}$ Rapport Tremblay, I: 213.

24 Ibid.

25 Ibid., 287.

26 Ibid., II : 80. 
du principe des nationalités “... compris dans le sens que chaque groupe national doit se constituer en Etat séparé" ${ }^{27}$. Il découle de cette analyse que le fédéralisme est le seul système qui peut permettre à deux cultures de vivre en paix et que le séparatisme n'est pas souhaitable.

Leur conception du Canada français et du fédéralisme implique que la province de Québec a des motifs propres de préserver son autonomie parce qu'elle est “... constituée par l'histoire foyer national, et par le droit, milieu politique fondamental du Canada français, elle est, comme unité politique, investie d'une mission dont aucune autre province n'a l'équivalent" ${ }^{28}$. On ajoute ensuite que cette conception autonomiste écarte le séparatisme et l'unitarisme. Cette "profession de foi" dans le fédéralisme a été faite, selon les auteurs, après une étude approfondie de la question et une enquête prolongée auprès des citoyens du Québec de toutes classes et de toutes origines ethniques. On y lit: "Les habitants du Québec, dans leur ensemble, ne désirent pas se retirer de la Confédération. Aucun des quelque 250 mémoires que notre Commission a reçus n'exprime un tel désir." ${ }^{29}$

Dans les derniers chapitres de la quatrième partie, on analyse le "nouveau fédéralisme", celui qui découlerait d'une mise en œuvre du Rapport Rowell-Sirois et des recommandations de la Commission Massey. Faisant, entre autres, la critique de la thèse centralisatrice, les auteurs écrivent:

Or la centralisation menée au nom de théories économiques et en vue d'objectifs avant tout économiques ne peut aboutir qu'à des résultats purement économiques, c'est-à-dire au sacrifice de la vie politique des citoyens devenus une masse mouvante de producteurs et de consommateurs. En fin de compte, c'est l'Etat lui-même qui perdra son caractère politique: socialistes, marxistes, communistes l'ont prédit d'ailleurs depuis longtemps par la voix d'Engels et de Lénine... ${ }^{30}$

On retrouve dans cette citation la peur morbide du socialisme et de l'emprise étatique qui se manifeste tout au long du Rapport. Cette phobie, même si elle s'explique historiquement, n'en demeure pas moins une faiblesse majeure de ce document. Cependant, lorsqu'il parle du comportement du gouvernement fédéral et des dangers que sa politique centralisatrice entraîne pour la survivance des Canadiens français, le Rapport a souvent

27 Ibid., II : 24 .

28 Ibid., II : 85.

29 Ibid., II : 90.

30 Ibid., II : 276-277. 
des phrases empreintes de lucidité, telle celle-ci qui fait la part de la dialectique majorité-minorité au sein du fédéralisme canadien:

Qu'on nous comprenne bien: il ne s'agit pas d'attribuer au gouvernement fédéral des sentiments d'hostilité à l'égard de la nationalité canadienne-française. Il s'agit plutôt de savoir si ce dernier, quand il légifère, peut faire autrement que de s'inspirer de la mentalité, des besoins et de la philosophie de la vie de la majorité anglo-protestante. Peut-il vraiment tenir une autre conduite ou chercher ailleurs son inspiration en régime démocratique? Mais alors, comment pourra-t-il adapter sa législation à une mentalité, à une culture et à des institutions différentes? De quelque côté qu'on l'envisage, la centralisation législative ne peut vouloir dire que nivellement, uniformisation et donc affaiblissement et ruine des valeurs nationales propres au groupe canadien-français. ${ }^{31}$

\section{Analyse des besoins et recommandations}

Dans la dernière partie du Rapport, les commissaires soulignent que les divers chapitres de cette partie sont l'application concrète des positions de principes des sections antérieures. Elle se divise en trois: les juridictions provinciales; les relations provinciales-municipales-scolaires; les relations fiscales et financières. Elle est basée, en majeure partie, sur les mémoires et les annexes soumis à la Commission. Elle envisage les ressources naturelles et l'aménagement du territoire, l'expansion démographique et le peuplement, l'organisation rurale, le bien-être et la santé, la sécurité sociale, l'enseignement, les œuvres de culture, les affaires municipales, et enfin le problème fiscal.

Modestement, la Commission souligne que ces études ne sont pas définitives et qu'elles doivent être poursuivies. Elle ajoute: "Les autorités politiques ont besoin, pour définir leurs objectifs et orienter leurs initiatives, d'une information étendue et sans cesse maintenue à jour, que seuls des services de recherches spécifiquement organisés peuvent leur assurer." 32

$\mathrm{Au}$ chapitre des juridictions provinciales, les sections consacrées au bien-être et à la santé, à la sécurité sociale et à l'enseignement, sont particulièrement intéressantes.

Bien-être, santé, sécurité sociale

Après avoir dressé un inventaire des œuvres de bien-être et de santé au Québec, on affirme: "Grâce à un ensemble un peu

31 Ibid., II : 280.

32 Ibid., III, 2 : 297. 
disparate d'initiatives, la province de Québec n'est pas en retard dans le domaine de la sécurité sociale" ${ }^{33}$, ce qui à notre avis, à la lecture même du Rapport, apparaît pour le moins présomptueux comme affirmation.

Cette section, qui est en grande partie basée sur l'annexe du père Gonzalve Poulin: L'Assistance sociale dans la province de Québec, 1608-1951, s'avère très conservatrice. On y manifeste une fois de plus une crainte extrême du socialisme en disant qu'une sécurité sociale, qui serait universelle, obligatoire, générale, distributive, gratuite, étatisée, serait d'inspiration socialiste, donc néfaste pour le Québec. On insiste sur la tradition, la famille, le clergé, pour assumer l'aide aux défavorisés.

L'incidence constitutionnelle de cette philosophie débouche sur des positions intéressantes, tirées en majeure partie de l'annexe 3 de François-Albert Angers: La sécurité sociale et les problèmes constitutionnels. On en vient à la conclusion que la province doit administrer elle-même sa propre juridiction sociale et utiliser à cette fin ses pouvoirs fiscaux. C'est le retour de la sécurité sociale aux provinces qui est préconisé, proposition dont on devait entendre parler très fréquemmment par la suite ! Mais il est amusant de penser que cette demande se faisait en partie en vue d'éviter au Québec un régime étatisé et socialisant de sécurité sociale!

\section{L'enseignement}

L'enseignement qui fait l'objet du chapitre suivant avait été étudié par Arthur Tremblay. Il s'était occupé de synthétiser les 140 mémoires qui traitèrent en tout ou en partie de l'enseignement. Il est intéressant de lire l'annexe rédigée par Arthur Tremblay en parallèle avec le Rapport. Tremblay n'est pas tendre pour le système scolaire, alors en vigueur au Québec, qu'il met doucement en pièces. Le tableau qui en résulte, que ce soit sur l'accessibilité aux études supérieures, sur la fréquentation scolaire, sur le financement des universités, ou sur la formation des maîtres, est très sombre. Il y est proposé une intervention beaucoup plus grande de l'Etat dans tous les domaines. Le Rapport atténue beaucoup certains jugements de l'annexe. D'ailleurs, les membres de la Commission avouent eux-mêmes avoir trouvé Arthur Tremblay assez audacieux dans ses propositions. "Il eut moins d'influence que certains experts. Nous l'avons consulté, mais nous le trouvions plus théoricien que pratique", nous a dit

33 Ibid., III, 1 : 128. 
le juge Tremblay. François-Albert Angers ajoute que la section sur l'éducation est ce qui lui a le moins plu dans le Rapport.

Il n'en demeure pas moins que le document final reprend de grandes parties de l'annexe et qu'il fait sienne la conclusion de ce travail lorsqu'il recommande au gouvernement de la province “... de faire continuer l'étude, par les moyens qu'il jugera lui-même appropriés; peut-être une nouvelle enquête confiée à des spécialistes, et ayant l'enseignement pour objet propre" ${ }^{34}$. Nous savons la suite de l'histoire. Particulièrement, la création de la Commission Parent et le rôle joué par ce même Arthur Tremblay dans la réforme de l'éducation au cours des années ' 60 .

\section{Le problème fiscal}

Une analyse des besoins financiers de la province et des municipalités faisant suite aux affirmations répétées du besoin d'autonomie pour le Québec débouche comme il se doit sur le problème fiscal.

Dans cette section, on rappelle d'abord les différents projets de règlements fiscaux proposés au cours des années précédentes. Mais selon la Commission ces projets ne correspondent pas aux dimensions réelles du problème. Il ne suffit pas de se procurer des fonds par la fiscalité. "Il importe en tout premier lieu, que par son inspiration et ses modalités, elle soit en accord avec la philosophie politique dont cet Etat est né et dont il doit continuer de vivre." 35

A partir de ces principes, la Commission expose les données maîtresses d'un réaménagement fiscal. Celui-ci s'établirait comme suit:

a) Au gouvernement fédéral: les impôts indirects et les impôts à incidence économique dont la pratique au palier des régions ou des localités tend à susciter des frontières à l'intérieur du pays: taxe de vente, taxes sur les divertissements et spectacles, sur l'essence, le tabac, les transferts de valeurs mobilières, etc.

b) Aux gouvernements provinciaux (outre la taxe foncière dévolue aux municipalités, les licences et permis, les redevances sur les ressources naturelles, les profits des entreprises étatisées), les impôts sur le revenu des particuliers et des compa-

\footnotetext{
34 Ibid., III, 1: 148.
}

35 Ibid., III, 2 : 228. 
gnies et l'impôt sur les successions. Bref, 100/100/100, c'est-à-dire la totalité des trois grands impôts directs.

Notons que ces propositions avaient été préparées par François-Albert Angers et exposées dans l'annexe 5: Le problème fiscal et les relations fédérales-provinciales. Parlant de l'influence de Angers sur la Commission, le juge Tremblay affirme: "Je trouvais qu'il était un peu trop volubile, mais il avait d'excellentes idées. Il nous a beaucoup aidés. L’idée du 100/100/100 est de lui. La réaction des membres de la Commission fut excellente, car nous pensions qu'il fallait poser le problème et nul ne savait si cette proposition n'allait pas se réaliser un jour." Angers de son côté avoue que ses idées ont surpris la Commission au début, mais qu'il n'y eut pas de forte résistance.

Ce plan se voulait un retour intégral à la constitution en matière sociale et fiscale. Mais, à notre avis, c'était un plan dangereux. Car, on ne sait jamais comment évoluera l'économie d'un pays. Evacuer complètement certains champs de taxation peut, à long terme, s'avérer une décision coûteuse.

D'aucuns ont porté sur ce Rapport un jugement plutôt sévère. Ainsi, on peut lire dans un récent manuel d'histoire que dans le Rapport Tremblay, "sous le couvert de l'autonomie, on cherchait à conserver les valeurs traditionnelles véhiculées par la petite-bourgeoisie traditionnelle canadienne-française". ${ }^{36} \mathrm{De}$ même, Michel Brunet écrivait il y a quelques années: "Le rapport Tremblay demeure la somme du nationalisme traditionnel avec toutes ses illusions et toutes ses contradictions." 37 A ce titre d'ailleurs, il s'agit d'un document important pour comprendre le Québec de l'après-guerre et en particulier le mouvement nationaliste de cette période qu'on aurait avantage à étudier avec autant de soin que celui du début du vingtième siècle, car il a joué dans notre histoire un rôle aussi important sinon plus que celui où se sont illustrés les Bourassa, les Asselin, et les LaVergne.

Quoi qu'il en soit, il serait difficile de rejeter le bien-fondé de ces critiques sur le conservatisme de ce document. Même si nous avons montré que la conception du fédéralisme canadien qu'on trouve dans le Rapport est tout à fait utopique, que sa peur de l'Etat est réactionnaire, que sa recommandation sur le partage des impôts est très discutable, il n'en demeure pas moins 586.

${ }^{36}$ Rosario Bilodeau et al., Histoire des Canadas (Montréal, 1971), 1958), 264. 
que son insistance sur le développement de la recherche, sa mise à jour des besoins du Québec, son argumentation fondée non seulement sur des principes mais sur des données économiques sont des aspects très positifs. Même si les commissaires ont pu errer sur bien des points, ils avaient raison sur l'essentiel, c'est-à-dire que l'autonomie provinciale était une condition vitale à la survie de la collectivité québécoise. D'ailleurs, un rédacteur du Financial Post avait bien vu, dès cette époque, l'importance idéologique du Rapport:

Up to the publication of this report, leaders of French Canadian nationalism had expressed their thoughts individually, unofficially, in an haphazard way. For the first time, they have had the opportunity of formulating their most extreme theories in many important fields through a commission in a publication officially sponsored by the Quebec Government. That fact in itself, constitutes a major victory for them. ${ }^{38}$

\section{La remise du Rapport}

Le 15 février 1956, le Rapport Tremblay fut remis à Duplessis. A l'encontre de ce qui se fait habituellement en de telles occasions, il n'y eut rien d'officiel. "Ce fut fait très simplement, commente le juge Tremblay; nous lui avons envoyé porter le paquet." Ce 'paquet' allait au cours des prochains mois, provoquer des épisodes dont le moins qu'on puisse dire est qu'ils tiennent à la fois de la tragi-comédie et du roman policier.

Le Rapport fut gardé secret, ce qui intrigua fortement tout le monde. Enfin, le 16 mars 1956, un mois après la remise du document, Duplessis annonça qu'il serait rendu public dans dix jours. ${ }^{39}$ Puis il partit en vacances. Le vendredi 6 avril, trois jours après le retour des Bahamas du premier ministre, paraissait un résumé du Rapport qui avait été remis à la Presse canadienne et reproduit dans la plupart des journaux le samedi 7 avril.

Ce jour-là, Le Devoir titrait en page neuf: "Le Rapport Tremblay suggère des remèdes radicaux" et étalait sur une pleine page les articles de la Presse canadienne de même que le compte rendu d'une conférence de presse donnée par Duplessis et à laquelle Le Devoir n'avait pas été convié. Le premier ministre affirmait "qu'il étudierait le Rapport en s'inspirant des faits" et ajoutait: "Si les Pères de la Confédération étaient honnêtes, lorsqu'ils ont institué des gouvernements provinciaux, ils doi-

38 "Radical tax change proposed in Québec's Tremblay Report", Financial Post (14 avril 1956) : 9.

39 Le Devoir, 16 mars 1956. 
vent alors avoir prévu certaines sources de revenus pour aider les mêmes gouvernements à s'acquitter de leurs obligations. Un tamisage s'impose pour transposer de la théorie à la pratique." 40

Le 9 avril, dans son bloc-notes, André Laurendeau écrivait:

... d'après les rapports d'agences, il contient des propositions révolutionnaires. $\mathrm{La}$ Commission propose de rendre aux provinces les impôts directs, et toutes les responsabilités sociales. En d'autres termes, c'est un retour intégral à la Constitution, après quarante ans d'évolution dans un sens centralisateur. (...) Mais il paraît trop radical pour risquer de sombrer dans l'indifférence. (...) Nous souhaitons qu'on prenne le moyen de le faire connaître dans les autres provinces. ${ }^{41}$

De leur côté, le Congrès des Métiers et du Travail et le Congrès canadien du Travail affirmaient, toujours en se fiant sur le résumé déjà paru, que le Rapport était totalement irréalisable. Claude Jodoin, président du CMTC et Donald Macdonald, secrétaire-trésorier du CCT, qualifiaient le document de "révolutionnaire". Ils exprimaient leurs craintes touchant la sécurité sociale qui serait laissée aux provinces si les recommandations étaient appliquées. ${ }^{42}$

Mais il demeurait impossible d'obtenir copie du Rapport. Le 16 avril, un Laurendeau désenchanté titrait son bloc-notes, "Un fiasco volontaire ?" et écrivait:

Les relations entre la presse et la Commission Tremblay ont été excellentes aussi longtemps que celle-ci a dirigé ses propres affaires. Elles se sont gâtées en dernière heure, c'est-àdire quand M. Duplessis les a prises en mains. Le lancement du rapport Tremblay est en effet un fiasco - et semble-t-il, un fiasco concerté. D'abord le Premier ministre l'a maintenu par-devers lui durant des semaines. Nous ne croyons pas que c'était pour en prendre connaissance... Puis les conclusions du Rapport ont été lancées dans le public il y a dix jours. Il semble que la divulgation ait été faite à quelques journalistes privilégiés. En tout cas nous n'avons pas été invités à cette cérémonie clandestine. ${ }^{43}$

Laurendeau continuait en ajoutant que toutes les démarches pour en obtenir un exemplaire avaient été inutiles.

Pourquoi cette pénombre? Pourquoi cette demi-publication, fort injuste pour tout le monde. Pourquoi ce faux lancement,

40 Ibid., 7 avril 1956.

41 Ibid., 9 avril 1956.

42 Ibid., 12 avril 1956.

43 Ibid., 16 avril 1956. 
qui met les gens en appétit, puis laisse l'intérêt tomber et la curiosité se diriger d'un autre côté ? Car le Rapport est publié. Il en existe au moins 3,000 exemplaires. Pourquoi deviennentils secret d'Etat ? (...) Une fois de plus, M. Duplessis oublie que nous vivons en démocratie parlementaire. (...) C'est une autre manifestation de son esprit possessif et tyrannique.44

Le silence se poursuivant, Gérard Filion, dans son éditorial du samedi 21 avril, lâchait le grand mot de "clandestinité" et s'exprimait en des phrases pouvant laisser croire que le Québec vivait sous un régime totalitaire !

Les trois mille exemplaires sortis de l'imprimerie depuis presque deux mois sont sous bonne surveillance de la police provinciale. (...) Les quelques journaux qui en possèdent un exemplaire l'ont obtenu comme Le Devoir, en contrebande. ${ }^{45}$

Devant cette attitude de Duplessis, l'on reste songeur. Qu'est-ce qui motiva un tel enterrement de première classe du Rapport ? Duplessis, au fond, n'avait-il voulu que "mollement cette enquête", comme l'avait déjà affirmé L'Action nationale.46

Nous avons interrogé nos témoins là-dessus. Le juge Tremblay de son côté croit qu'au début Duplessis voulait la Commission. "Mais au milieu, à la fin surtout, son attitude se modifia. Il fut surpris. Il croyait que nous publierions un petit rapport à l'eau de rose comme ses discours à Ottawa. C'est peut-être la raison pour laquelle le Rapport est demeuré sur les tablettes." Il poursuit:

Il est certain qu'il ne l'a jamais lu. C'était un homme extrêmement méfiant. Il a toujours pensé que le problème n'était pas compliqué. Ce fut le silence complet: pas de compliments, pas de reproches. Je l'ai rencontré plusieurs fois au cours de notre enquête. Il trouvait qu'on compliquait trop notre travail. Il disait: "Si je le faisais votre rapport, ce ne serait pas long !"

Jean-Charles Bonenfant donne la version suivante: "Duplessis a accepté la Commission pour se débarrasser des nationalistes de Montréal. Lui, était un nationaliste traditionnel. Le Rapport pose un début de nationalisme canadien-français positif." Quant à la fausse sortie du Rapport, il ajoute: "C'était un homme vieillissant, incapable d'en voir la portée. Alors, il préféra le cacher. Je pense que toute l'explication vient du fait qu'on est, en 1956 , trois ans avant sa mort."

44 Ibid.

45 Ibid., 21 avril 1956.

46 L'Action nationale, 45 (mai 1956) : 771. 
Pour Paul-Henri Guimont, la réception faite au Rapport était un peu prévue.

On s'en doutait de plus en plus. Le juge avait de moins en moins de contacts. Un facteur me laissa perplexe: lorsque je fis le tour des ministères pour demander aux sous-ministres des rapports sur les activités de leur ministère, je n'obtins rien là où des amis de Duplessis étaient en place. Ainsi, pendant six mois, je tentai d'obtenir un rapport de Desaulniers (surintendant de l'Instruction publique) sans succès. Pour moi, Duplessis était un politicien qui avait gagné sa cause avec l'Impôt. Il était physiquement diminué. Il n'avait ni la capacité physique, ni la capacité mentale de mettre en œuvre un tel programme qui l'entraînerait dans un dédale d'aventures doit il ne pourrait sortir. dit :

François-Albert Angers voit les choses autrement quand il

Ce n'est pas le vieillissement, c'est lui ! Il avait l'impression qu'il n'avait plus grand'chose à faire. Ce qui ne l'incitait pas à agir d'autre part. Duplessis ne voulait pas un Rapport comme ça. Il aurait préféré une Commission qui aurait travaillé trois ou quatre mois. Eux en ont profité pour faire une immense Bible. Il n'a pas prisé la chose. Ces gens se mêlaient de ses affaires et risquaient de lui proposer des choses qu'il ne voulait et ne pouvait pas entreprendre. Il ne l'a pas lu. Attitude de politicien. Il n'était pas pour s'engager à ça. Alors, en somme, il a dit "C'est un beau Rapport. Il faudra le faire examiner par des gens pratiques."

N'avait-il pas dit, d'ailleurs, lors de la conférence de presse du 6 avril: "Le problème constitutionnel n'est pas compliqué en réalité. Il s'agit simplement de savoir si les provinces auront l'argent nécessaire pour rencontrer leurs obligations constitutionnelles." 47

Monsieur Roger Stanton mentionne aussi que Duplessis aurait eu une certaine appréhension de la réaction des anglophones. On peut comprendre ceci lorsqu'on lit certaines critiques du Rapport dans les publications anglophones. Ainsi, dans le numéro de juin du journal The Canadian Forum, Martin P. O'Connell écrivait:

The Report unexpectedly threw nationalism on the defensive. It wrote into an official document a clear statement of principles and aims and, in the name of the "spirit of federalism", it proposed an extravagant reshaping of fiscal structures which would involve such wrenching of the fed-

${ }^{47}$ Le Devoir, 7 avril 1956. 
eral system that few responsible leaders are likely to support it.

Parlant de la réponse unanime de la population à l'enquête, il ajoute:

This is perhaps more than even M. Duplessis bargained for if it means, as it seems to mean, that the Commission's recommendations are the unanimous views of M. Duplessis' electorate. ${ }^{48}$

Chose certaine, Duplessis n'aimait guère le Rapport où pourtant avec un peu d'imagination il aurait pu reconnaître les bases sur lesquelles sa pensée autonomiste reposait.

\section{Une mise à l'index profitable}

Tout contribuable pouvait consulter ledit rapport à la bibliothèque de l'Assemblée. $\mathrm{A}$ défaut de pouvoir se rendre à Québec pour quelques jours, il lui était possible d'écrire à monsieur JeanCharles Bonenfant et de lui demander, comme faveur ou presque, une copie du document. Comme si ce n'était pas suffisant pour limiter la diffusion du Rapport, très tôt, au témoignage même de Bonenfant, on l'a surveillé et il devait envoyer les demandes chez le premier ministre. Inutile de dire qu'en 1960 la première édition de 3,000 exemplaires n'était pas encore épuisée !

Après coup, il semble évident que cette mise à l'index loufoque du Rapport lui fut profitable. A ce sujet, Angers se prononce catégoriquement.

Si Duplessis avait adopté le Rapport, l'opposition l'aurait démoli. Cette attitude a fait du Rapport un document fondamental, une Bible des Canadiens français. Une commission royale a établi la base de toute notre survivance. C'est un document approuvé par le gouvernement (au départ), et repris par les Libéraux. J'ai l'impression que Duplessis a rendu ainsi un fier service à la province. Les cohortes du père Lévesque seraient sorties pour le démolir s'il l'avait approuvé.

Le père Arès abonde dans le même sens lorsqu'il dit: "Le Rapport, largement diffusé, aurait été difficilement digéré, car à cette époque, l'intelligentsia vomissait tout ce qui venait de Duplessis. Quand on vit que Duplessis le retenait, il (le Rapport) s'acquit une certaine sympathie." Effectivement, le Rapport

48 Martin P. O'Connell, "M. Duplessis' Royal Commission", Canadian Forum, 36 (juin 1956) : 50-52. 
devait, malgré et peut-être à cause de sa semi-clandestinité, porter des fruits. Cela est si vrai qu'on a pu lire à l'article $41 \mathrm{du}$ programme du parti libéral en 1960: "Présentation par la province d'un mémoire devant la Conférence interprovinciale pour la solution du problème fiscal, le rapport de la Commission Tremblay devant servir de base à ce mémoire". ${ }^{49}$ De plus, lors de la conférence fédérale-provinciale de juillet 1960, Lesage fraîchement élu, avait apporté dans ses bagages le Rapport Tremblay qu'il déposa à la table des négociations. ${ }^{50}$

Sans refuser toute valeur au Rapport, ce qui nous paraît le plus important, c'est le rôle joué par la Commission.

Nous avons mentionné l'influence déterminante des commissaires et, en particulier du juge Tremblay, dans la création d'un impôt provincial sur le revenu en 1954. Cette mesure, à notre avis, a constitué un point tournant dans l'évolution du Québec. Le juge Tremblay et ses collègues ont pu jouer ce rôle décisif parce qu'ils ont parcouru la province, reçu des centaines de mémoires, entendu des centaines de personnes et ainsi acquérir la conviction que la population appuierait Duplessis s'il osait agir de manière unilatérale.

Les recherches que la Commission suscita, la prise de conscience que de nombreux groupes firent à cette occasion, les lacunes qu'elle mit à jour, la solidarité qu'elle révéla vis-à-vis de l'Etat québécois font de cette Commission un événement majeur de l'histoire du Québec.

D'une certaine manière, nous pourrions dire comme Marshall McLuhan: "Societies have always been shaped more by the nature of the media by which men communicate than by the content of communication." ${ }^{51}$ En d'autres mots, dans le cas de la Commission Tremblay, le médium a été plus important que le message.

II : 252-253.

49 Michel Brunet, Histoire du Canada par les textes (Montréal, 1963),

51 Marshall McLuhan, The Medium is the Massage (Toronto, 1967), 8.

so Ibid., 249. 